$\Rightarrow$ TRANSCRIPTION FACTORS

\section{MYC matters}

Mutation or aberrant regulation of the transcription factor MYC is a common feature of many cancers. Two new studies have added more details to the complexity of MYC regulation in cell development and growth pathways, underscoring the importance of MYC in tumorigenesis.

Transcriptional profiling has recently revealed that embryonic stem (ES) cells and cancer cells share common expression signatures. Does this reflect underlying similarities between ES cells and cancer cells? And what role does MYC have in these similarities? To address these questions, Kim et al. first characterized a MYC-centred protein interaction network in ES cells, and found that this network did not overlap with a protein interaction network centred on core pluripotency factors that had been defined in ES cells in another study. The authors then used chromatin immunoprecipitation (ChIP) to define the genomic targets of MYC, and integrated this data with previously published ChIP-based data sets to obtain a comprehensive picture of the genes that are bound by key transcription factors. From

the MYC-regulated network is responsible for the similarities between ES cells and cancer cells.
Kim et al. then used these target gene modules to see how ES cell signatures relate to expression changes in cancers in mice and humans: they found that the Core module was not significantly expressed in these cancers, but that the Myc module was highly expressed, suggesting that the MYC-regulated network is responsible for the similarities between ES cells and cancer cells. Moreover, activity of the Myc module could be used as a prognostic indicator in human breast cancer samples, with high Myc module activity predicting faster metastasis. Importantly, these findings refute the hypothesis that cancer development requires the reactivation of ES cell pluripotency programmes, and highlight the key role of MYC in both ES cell and cancer cell control networks.

In a second paper, Laura Johnston and colleagues uncover a relationship between MYC and the Hippo tumour suppressor pathway that is important in growth regulation. They investigated the effect of Yorkie (YKI) (the Drosophila melanogaster homologue of the transcriptional co-activator YAP, which is normally inhibited by Hippo signalling) in growth control of D. melanogaster imaginal discs. They found that YKI binds to the gene encoding MYC $(\mathrm{dm})$ and regulates its transcription. Moreover, MYC can regulate YKI expression by both transcriptional and post-transcriptional mechanisms, allowing MYC to indirectly regulate its own expression. This also provides a mechanism to balance the growth-promoting activities of

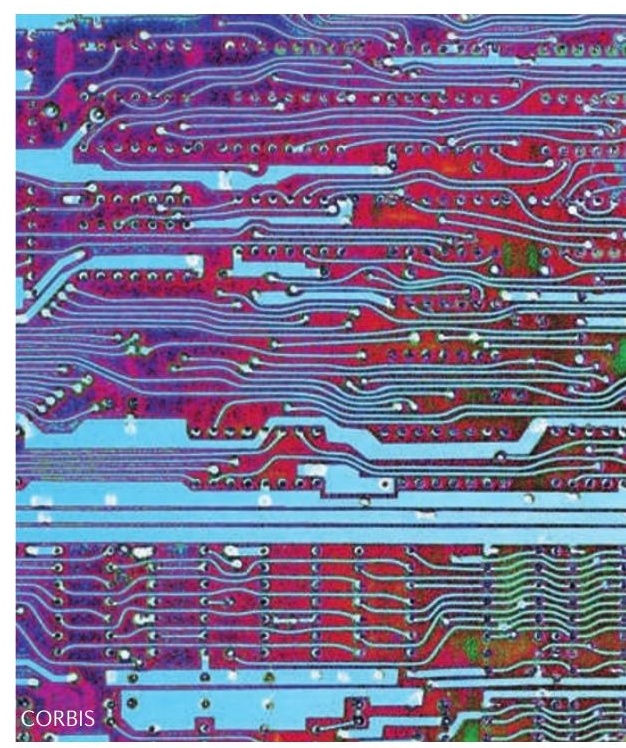

MYC and the growth suppression that is mediated by the Hippo pathway. Interestingly, the authors found that co-expression of MYC and YKI led to significantly more disc growth than the expression of either factor alone, which was consistent with previous findings from mouse models that YAP and MYC can synergize to accelerate tumour formation. Amplification of 11q22, which contains YAP, has been observed in several human cancers, raising the possibility that YAP deregulation of MYC might be important during tumorigenesis. Therefore, this study has identified a novel regulatory relationship that deserves further investigation in humans for its potential role in cancer.

Meera Swami

ORIGINAL RESEARCH PAPERS Kim, J. et al. A Myc network accounts for similarities between embryonic stem and cancer cell transcription programs. Cell 143, 313-324 (2010)| Neto-Silva, R. M., de Beco, S. \& Johnston, L. A. Evidence for a growth-stabilizing regulatory feedback mechanism between Myc and Yorkie. the Drosophila homolog of Yap. Dev. Cell 19, 507-520 (2010) 